\title{
Chitosan/bioactive glass nanoparticle composite membranes for periodontal regeneration
}

\author{
Joana Mota a,b ${ }^{\mathrm{a}}$ Na Yu ${ }^{\mathrm{c}}$, Sofia G. Caridade ${ }^{\mathrm{a}, \mathrm{b}}$, Gisela M. Luz ${ }^{\mathrm{a}, \mathrm{b}}$, Manuela E. Gomes ${ }^{\mathrm{a}, \mathrm{b}}$, Rui L. Reis ${ }^{\mathrm{a}, \mathrm{b}}$, \\ John A. Jansen ${ }^{c}$, X. Frank Walboomers ${ }^{c}$, João F. Mano ${ }^{\mathrm{a}, \mathrm{b}, *}$ \\ a 3B's Research Group - Biomaterials, Biodegradables and Biomimetics, University of Minho, Headquarters of the European Institute of Excellence on Tissue Engineering \\ and Regenerative Medicine, AvePark, 4806-909 Taipas, Guimarães, Portugal \\ ${ }^{\mathrm{b}}$ ICVS/3B's - PT Government Associate Laboratory, Braga/Guimarães, Portugal \\ ${ }^{\mathrm{c}}$ Radboud University Nijmegen Medical Centre, Department of Biomaterials, P.O. Box 9101, 6500HB Nijmegen, The Netherlands
}

\section{A R T I C L E I N F O}

\section{Article history:}

Received 13 April 2012

Received in revised form 27 June 2012

Accepted 29 June 2012

Available online 5 July 2012

\section{Keywords:}

Biomaterials

Tissue engineering

Guided tissue regeneration

Chitosan

Nanoparticles

\begin{abstract}
A B S T R A C T
Barrier membranes are used in periodontal applications with the aim of supporting periodontal regeneration by physically blocking migration of epithelial cells. The present work proposes a combination of chitosan (CHT) with bioactive glass nanoparticles (BG-NPs) in order to produce a novel guided tissue and bone regeneration membrane, fabricated by solvent casting. The CHT/BG-NP nanocomposite membranes are characterized in terms of water uptake, in mechanical tests, under simulated physiological conditions and in in vitro bioactivity tests. The addition of BG-NPs to CHT membranes decreased the mechanical potential of these membranes, but on the other hand the bioactivity improved. The membranes containing the BG-NPs induced the precipitation of bone-like apatite in simulated body fluid (SBF). Biological tests were carried out using human periodontal ligament cells and human bone marrow stromal cells. CHT/BG-NP composite membranes promoted cell metabolic activity and mineralization. The results indicate that the CHT/BG-NP composite membrane could potentially be used as a temporary guided tissue regeneration membrane in periodontal regeneration, with the possibility to induce bone regeneration.
\end{abstract}

(c) 2012 Acta Materialia Inc. Published by Elsevier Ltd. All rights reserved.

\section{Introduction}

Periodontitis is an inflammatory disease of the periodontal tissues, caused by microorganisms and calculus accumulation on the bacterial biofilm, leading to degradation of the connective tissues and alveolar bone and subsequent formation of soft tissue pockets around the root surface [1]. Regeneration of the periodontal region is challenged by complex inflammatory processes and events related to healing, resulting in unsatisfactory results in clinical cases using the currently available therapies. Ideal strategies should be able to regenerate all damaged structures including the cementum, periodontal ligament and alveolar bone. The number of cells occupying the treated area upon surgery as well as the size of the defect determine the type of connection formed between the cementum and bone. Epithelial cells are the first cells to migrate to the site of injury becoming a problem as they prevent bone formation.

\footnotetext{
* Corresponding author at: 3B's Research Group - Biomaterials, Biodegradables and Biomimetics, University of Minho, Headquarters of the European Institute of Excellence on Tissue Engineering and Regenerative Medicine, AvePark, 4806-909 Taipas, Guimarães, Portugal. Tel.: +351 253510900; fax: +351 253510909.

E-mail address: jmano@dep.uminho.pt (J.F. Mano).
}

The guided tissue regeneration (GTR) technique uses a membrane, which acts as a barrier to prevent epithelial cells and gingival tissue reaching the area of injured tissue [2]. This procedure favors the regeneration of lost and damaged tissue, as it promotes cell repopulation of the periodontal ligament and adjacent alveolar bone, allowing the necessary time for osteoblast proliferation and bone regeneration $[3,4]$. Cells derived from adult human bone marrow stromal cells (hBMSC) are capable of contributing to the formation of new bone completely surrounding the tooth, which has to be able to anchor itself to the jaw through its roots and periodontal ligament [5]. The continuing process of periodontal tissue repair is followed by the formation of granulation tissue as a source of future periodontal connective tissue cells, such as osteoblasts, periodontal ligament fibroblasts and cementoblasts [5]. Thus human periodontal ligament cells (hPDL) and hBMSCs are ideal cell sources to be used in biological tests to evaluate the performance of GTR membranes.

When selecting an ideal biomaterial for GTR membranes the following requirements must be considered: wound stabilization, space creation and maintenance, protection of the underlying blood clot, and the ability to exclude unwanted tissues or cells (connective tissue and gingival epithelium). Collagen has been 
one of the most common materials used to produce GTR membranes. However, this material is derived from animal sources and has an associated risk of disease transmission and associated ethical and cultural issues. Moreover, the fast resorption rate of this material and its poor mechanical strength are a concern to many clinicians. In addition, rapid collagen biodegradation, by the enzymatic activity of macrophages, polymorphonuclear leucocytes and several pathogens capable of producing collagenase, results in a limited membrane resistance to collapse. This event allows unwanted cell types to enter the wound area [6]. Since collagen membranes have shown such limited properties, new materials with better properties are required.

Chitosan (CHT) is obtained by alkaline deacetylation of chitin and presents excellent biological properties, such as biodegradability, biocompatibility, and immunogenicity, as well as antibacterial, antifungal and wound-healing activity. Its degradation products are non-toxic, non-antigenic, non-immunogenic and non-carcinogenic. CHT also evokes minimal foreign body reaction [7-12]. The positive surface charge of this biomaterial and its biocompatibility enable it to effectively support cell growth, while the hydrophilic surface facilitates cell adhesion, proliferation, and differentiation $[7,11,13]$. When placed in hydrated environments CHT is a flexible material. This is an advantage over more rigid synthetic materials like polylactic acid (PLA) and polyglycolic acid (PGA), facilitating handling during the implantation process [14-16]. Because of these properties CHT has been widely used in biomedical applications $[9,12,13,17]$. It can accelerate wound healing and enhance bone formation both in vivo and in vitro. It has been reported that when implanted in vertebrates it may enhance the migration and differentiation of specific types of progenitor cells (e.g. osteoblast differentiation) $[10,18,19]$. Lahiji et al. found that CHT may serve as an effective template in the repair of osseous defects, because it has unique material properties and the ability to support viable and functioning human osteoblasts. [9] Thus CHT is an attractive candidate for future use in GTR membranes. However, CHT is not an ideal material for bone regeneration, as its osteoconductivity needs to be improved. Zhang et al. [20] verified that no apatite was formed on the surface of pure CHT scaffolds. To improve the bioactivity of CHT it is necessary to combine it with other bioactive materials [21]. Recent studies have highlighted the importance of the development of composite materials based on biodegradable polymers containing bioactive glasses [22]. Bioactive glasses often show excellent bioactivity and biocompatibility both in vitro and in vivo: when implanted at the site of bone defects they can directly bond to the surrounding tissues by chemical linkage. As well as the GTR function, acting as a barrier, the addition of bioactive glass particles to CHT membranes should promote guided bone regeneration (GBR) activity, giving a dual function, producing a GTR/GBR membrane. The biomineralization capability of bioactive glasses is related to its composition, but is also influenced by physical properties such as particle size, porosity, surface area and morphology. In recent years a growing consensus has been reached to concentrate on the development of nanostructural bioactive glass/ ceramic materials, due to their high biomineralization capability. Large surface area bioactive glass materials should facilitate bone healing and tooth defects, as well as blood clotting. Consequently, the preparation and application of nanoscale bioactive glass materials has received increasing attention from biological material researchers. The sol-gel method has been used to prepare high surface area bioactive glass particles, including bioactive glass nanoparticles (BG-NPs) [10,23-26].

In this study CHT/BG-NP nanocomposite membranes were prepared by solvent casting, and the physical, bioactive and biological properties of these biomaterials were characterized, to evaluate the possible performance in periodontal defect applications, using hPDL and hBMSC cells in biological tests.

\section{Materials and methods}

Chitosan (CHT) ( $\mathrm{M}_{\mathrm{w}}$ 190,000-310,000, 75-85\% deacetylated, viscosity $200-800 \mathrm{cps}$ ) was obtained from Sigma and was purified before use. Tetraethyl orthosilicate (TEO) (99.90\% pure), ammonium phosphate dibasic, calcium nitrate tetrahydrate (99\%), citric acid monohydrate (99-100\%), ammonium hydroxide (maximum $33 \% \mathrm{NH}_{3}$ ) and all chemicals for SBF preparation were purchased from Sigma-Aldrich. All other reagents and solvents used were of reagent grade.

\subsection{Bioactive glass nanoparticle preparation}

To prepare the BG-NPs a protocol based on previous work was followed $[27,28]$. The procedure to obtain nanoparticles with the composition $\mathrm{SiO}_{2}: \mathrm{CaO}: \mathrm{P}_{2} \mathrm{O}_{5}(\mathrm{~mol} . \%)=55: 40: 5$ consisted of sequential reagent dissolution that resulted in hydrolysis and polycondensation reactions. TEOS (99.90\% pure) was used as the silicon precursor, ammonium phosphate dibasic as the phosphorus precursor, calcium nitrate tetrahydrate (99\%) as the calcium precursor, citric acid monohydrate (99-100\%) to promote hydrolysis, absolute ethanol, ammonium hydroxide (maximum 33\% $\mathrm{NH}_{3}$ ) as the gelling agent and polyethylene glycol 20,000 (PEG) as the surfactant. The BG-NPs were sintered at $700{ }^{\circ} \mathrm{C}$ for $5 \mathrm{~h}$. Spherical particles with sizes below $50 \mathrm{~nm}$ were obtained (see Fig. 3).

\subsection{Membrane preparation}

Composite membranes were obtained by the dissolution of $0.7 \%$ $(\mathrm{w} / \mathrm{v})$ purified CHT and $0.3 \%(\mathrm{w} / \mathrm{v})$ BG-NPs in a solution of 2 vol.\% acid acetic. Pure CHT membranes were obtained by dissolution of $1 \%(\mathrm{w} / \mathrm{v})$ CHT in a solution of 2 vol.\% acid acetic. After complete solvent evaporation the membranes were neutralized with a solution of $0.4 \%(w / v) ~ N a O H$ and left to dry at room temperature.

\subsection{Water uptake}

$1 \times 1 \mathrm{~cm}$ samples cut from the membranes were weighted $\left(W_{\text {ini }}\right)$ and then immersed at $37^{\circ} \mathrm{C}$ in $2 \mathrm{ml}$ of phosphate-buffered saline (PBS), $\mathrm{pH} 7.4$, containing $0.8 \%(\mathrm{w} / \mathrm{v})$ lysozyme (hen eggwhite) and $0.02 \%(\mathrm{w} / \mathrm{v})$ sodium azide. The concentration of lysozyme was similar to the concentration in human serum [29]. Membranes of known dry weight ( $n=3$ samples of each set of membranes) were incubated in the lysozyme solution for the period of study. At each time point samples were removed from the solution and rinsed with distilled water. The superficial water was removed and samples were weighted $\left(W_{\text {fin }}\right)$. The water uptake was calculated using the equation:

$\left(W_{\text {fin }}-W_{\text {ini }}\right) / W_{\text {ini }} \times 100(\%)$

\subsection{Mechanical tests}

The ultimate tensile strength (UTS) and tensile modulus of the membranes were determined using an Instron 4505 Universal Machine tensile testing machine in tensile mode. The tests were performed at room temperature at a rate of $5 \mathrm{~mm} \mathrm{~min}^{-1}$. Wet membranes were immersed in PBS for $3 \mathrm{~h}$ before the test and were fixed with sandpaper, to prevent slipping. Tests were performed on a single membrane with a gauge length of $10 \mathrm{~mm}$. For each condition a minimum of five specimens were tested.

\subsection{In vitro bioactivity tests}

In vitro bioactivity tests were carried out by soaking the membranes in $50 \mathrm{ml}$ of SBF for 5 days at $37^{\circ} \mathrm{C}$. The SBF composition and 
preparation was previously described by Kokubo and Takadama [30]. Upon removal from the SBF the samples were rinsed with distilled water and left to dry.

\subsection{Scanning electron microscopy (SEM) and energy dispersive spectroscopy (EDX) experiments}

A NanoSEM FEI Nova 200 (FEG/SEM) scanning electron microscope was used to study the surface and the morphology of the samples. A conductive gold coating was applied to the samples prior to observation. A Pegasus X4M instrument was used to preform the energy dispersive X-ray spectroscopy (EDX) experiments, in a low vaccum and without coating.

\subsection{Cell culture}

Periodontal ligament cells were obtained from human third molars according to the following procedure. After extraction the teeth were washed three times for $10 \mathrm{~min}$ in PBS with 100 units $\mathrm{ml}^{-1}$ penicillin/streptomycin. Periodontal ligament tissue was scraped from the middle third of the root with a scalpel blade, to avoid contamination by epithelial or pulp cells. The freed portions of the periodontal ligament were minced and transferred to a small culture flask, which was filled with alpha minimal essential medium ( $\alpha$-MEM) (Gibco) containing 10 vol.\% fetal calf serum (FCS) (Gibco), $50 \mathrm{mg} \mathrm{ml}^{-1}$ ascorbic acid (Sigma), 10.8 M dexamethasone (Sigma), $50 \mathrm{mg} \mathrm{ml}^{-1}$ gentamycin (Gibco) and $10 \mathrm{mM}$ sodium $\beta$-glycerophosphate (Sigma). Cells were cultured at $37^{\circ} \mathrm{C}$ in a humidified atmosphere with $5 \% \mathrm{CO}_{2}$. The medium was replaced every $2-3$ days. Upon reaching $100 \%$ confluency the cells were released with trypsin/EDTA $(0.25 \% \mathrm{w} / \mathrm{v}$ crude trypsin and $1 \mathrm{mM}$ EDTA, pH 7.2) and sub-cultured for two passages in standard culture flasks. The cells were then frozen in liquid nitrogen until use.

hBMSCs were isolated from bone blocks of human iliac crest biopsies. The biopsies were discarded tissue from standard surgical procedures at Radboud University Nijmegen Medical Centre (Nijmegen, The Netherlands). The bone blocks were cut into small pieces and subsequently placed in a $50 \mathrm{ml}$ tube to which $20 \mathrm{ml}$ of $\alpha$-MEM was added. Then the tubes were shaken vigorously and the medium with cells collected. This procedure was repeated several times. The collected medium with cells was plated in T25 culture flasks (Greiner Bio-one), which were filled with $4 \mathrm{ml}$ of $\alpha$-MEM containing $10 \mathrm{vol} . \% \mathrm{FCS}$ and 100 units $\mathrm{ml}^{-1}$ penicillin/streptomycin (all from Gibco BRL Life Technologies BV, Breda, The Netherlands). The cells were cultured at $37{ }^{\circ} \mathrm{C}$ in a humid atmosphere with $5 \%$ $\mathrm{CO}_{2}$ and passaged at $80 \%$ confluency using trypsin/EDTA (Gibco).

Two kinds of proliferation medium were used (hereafter referred to as $\mathrm{O}^{-}$culture media). The hPDL $\mathrm{O}^{-}$culture medium was composed of $\alpha$-MEM (Gibco) with $10 \%$ fetal bovine serum (FBS) (Greiner Bio-one) and 100 units $\mathrm{ml}^{-1}$ penicillin/streptomycin (Gibco BRL). The hBMSC $\mathrm{O}^{-}$culture medium was composed of $\alpha$-MEM (Gibco) with 15\% FBS (Greiner Bio-one), 1\% L-glutamine, 1\% ascorbic acid (Sigma), 100 units $\mathrm{ml}^{-1}$ penicillin/streptomycin (Gibco BRL) and $1 \%$, by volume added to each cell culture flask, basic fibroblast growth factor (bFGF).

After one generation the cells were plated at a density of 5000 cells $\mathrm{cm}^{-2}$ in $\mathrm{T} 75$ culture flasks and expanded in $\mathrm{O}^{-}$culture medium. The culture medium was changed twice a week. Cells from passage 3 were used in this experiment.

\subsection{Cell seeding}

The membranes were glued to metal rings $(1.5 \mathrm{~cm}$ diameter) with RTV silicone adhesive (Nusil Technology, Carpinteria, CA), in order to prevent membrane fluctuation and to fix them on the culture well bottom. The samples were sterilized by immersion in $70 \%$ ethanol for $1 \mathrm{~h}$, rinsed in PBS and soaked in medium overnight. Cells, either hPDL or hBMSC, were seeded on the samples and after $4 \mathrm{~h}$ (at $37{ }^{\circ} \mathrm{C}$ and $5 \%$ of $\mathrm{CO}_{2}$ ) osteogenic differentiation culture medium $\left(\mathrm{O}^{+}\right.$culture medium) was added. As controls cells were seeded directly on the bottom of the plate and $\mathrm{O}^{+}$culture medium was added immediately.

Two types of $\mathrm{O}^{+}$culture medium were used. The hPDL $\mathrm{O}^{+}$culture medium was composed of $\alpha$-MEM (Gibco) with $10 \%$ FBS (Greiner Bio-one), $1 \%$ ascorbic acid (Sigma), 1\% $\beta$-glycerophosphate (Sigma), 1\% dexamethasone (Sigma) and 100 units $\mathrm{ml}^{-1}$ penicillin/streptomycin (Gibco BRL). The hBMSC $\mathrm{O}^{+}$culture medium was composed of $\alpha$-MEM (Gibco) with 15\% FBS (Greiner Bio-one), $1 \%$ L-glutamine, $1 \%$ ascorbic acid (Sigma), $1 \% \beta$-glycerophosphate (Sig$\mathrm{ma}$ ), $1 \%$ dexamethasone (Sigma) and 100 units $\mathrm{ml}^{-1}$ penicillin/ streptomycin (Gibco BRL).

\subsection{Biological tests}

\subsubsection{Immunofluorescence and image analyses}

After 3 days in culture on the membrane surface cells were fixed for $10 \mathrm{~min}$ in $3 \%$ paraformaldehyde (Fluka AG), and permeabilized with $1 \%$ Triton X-100 for 5 min. Then filamentous actin was stained with AlexaFluor 568 phalloidin (Molecular Probes Inc., Eugene, OR) diluted 1:200 in PBS containing $1 \%$ bovine serum albumin (BSA) for $2 \mathrm{~h}$. The cells nuclei were stained with 4,6diamidino-2phenylindole (DAPI) diluted $1: 2500$ in PBS for $10 \mathrm{~min}$. Finally, the specimens were examined with a Zeiss automated fluorescence microscope (Imager Z1) at a magnification of $10 \times$.

\subsubsection{Proliferation assay}

After the various experimental periods the medium was removed from the chambers and the wells were washed with PBS. The cells were lysed using milliQ filtered water with subsequent sonication for $10 \mathrm{~min}$ between two freeze-thaw cycles at $-80^{\circ} \mathrm{C}$. The supernatant was stored at $-20^{\circ} \mathrm{C}$ until further analysis. A PicoGreen dsDNA Quantification Kit (Molecular Probes, Eugene, OR) was used according to the manufacturer's instructions. The analysis was performed on the supernatants from days 1, 3, 7, 14 and 28. $100 \mu \mathrm{l}$ of PicoGreen working solution was added to $100 \mu \mathrm{l}$ of each supernatant sample. The samples were incubated for 2-5 min at room temperature in the dark. After incubation fluorescence was measured in a fluorescence cuvette reader (microplate fluorescence reader, Bio-Tek Instruments, Winooski, VT) with a $485 \mathrm{~nm}$ excitation filter and a $530 \mathrm{~nm}$ emission filter.

\subsubsection{Alamar blue}

Cell metabolic activity was measured using the Alamar blue assay (Invitrogen) according to the manufacturer's instructions. A solution was made of Alamar blue in culture medium in the proportions $1: 9$ and held at $37^{\circ} \mathrm{C}$ for $5 \mathrm{~min}$. Culture medium was flushed from wells containing samples with cells and replaced with the test solution. Culture plates were incubated at $37^{\circ} \mathrm{C}$ in $5 \% \mathrm{CO}_{2}$ for $4 \mathrm{~h}$. After incubation $200 \mu \mathrm{l}$ of each sample solution were transferred to 96-well plates (Greiner Bio-one). Fluorescence was measured using a microplate reader (FL 600, Bio-Tek Instruments) at $570 \mathrm{~nm}$. The assay was performed on days 1, 3, 7, 14 and 28.

\subsubsection{Calcium content measurement}

Calcium content was assessed 28 days after cell seeding to obtain information on mineralized matrix formation. The samples were rinsed with milliQ filtered water and $1 \mathrm{ml}$ of acetic acid was added. The samples were incubated overnight under vigorous constant shaking and the acetic acid with dissolved calcium was frozen and kept at $-20^{\circ} \mathrm{C}$ until further investigation. After thawing the calcium content was determined using the OCPC 
(o-cresolphthalein complex one) method. Optic density was read with an ELISA reader (Bio-Tek Instruments, Winooski, VT) at a wavelength of $570 \mathrm{~nm}$.

\subsection{Statistical analysis}

Every sample was measured in triplicate. Statistical analysis was performed using an unpaired ordinary ANOVA, using standard parametric methods, and Tukey's test. Calculations were performed in InStat (v.3.0 GraphPad Software Inc., San Diego, CA).

\section{Results and discussion}

\subsection{Water uptake}

Homogeneous membranes were produced using the solvent casting methodology described above. Water uptake measurement curves for the CHT/BG-NP composites and pure CHT membranes are shown in Fig. 1.

From Fig. 1 it can be seen that swelling reached equilibrium after $\sim 5 \mathrm{~min}$ immersion in the cocktail solution. The water uptake values are similar for both pure CHT and the CHT/BG-NP composite membranes, of the order of $130 \%$. This behavior is similar to the results found before for CHT membranes prepared by a similar methodology, in which swelling equilibrium took place after $\sim 15 \mathrm{~min}$ [31]. No significant changes in water uptake were detected over a period of 16 weeks, which is consistent with the stability of weight loss over 14 weeks, indicating slow degradation in vitro (data not shown). Ren et al. [32] obtained similar CHT water uptake values to this study of $\sim 130 \%$, without significant variations over time [32]. Peter et al. [33,34] measured the swelling of CHT and CHT with bioactive glass ceramic scaffolds in the same way as in this study. However, a significant reduction in water uptake was found on the addition of nanoparticles to the scaffolds at one time point $[33,34]$. In contrast, Maquet et al. $[35,36]$ showed that the addition of BG-NPs to a polymer increased water absorption.

\subsection{Tension tests}

Tensile testing under dry and wet conditions was conducted to evaluate the mechanical properties of both the CHT and CHT/BGNP composite membranes (see Fig. 2 and Table 1). Biomedical implants are usually placed in very hydrated environments, which may significantly influence their performance compared with the dry state. The mechanical behavior under wet conditions is very important in predicting the mechanical properties of membranes in vivo, which determine both their clinical operation and bone healing capacity $[37,38]$.

Representative stress-strain curves from tensile tests obtained for pure CHT and CHT/BG-NP composite membranes, under both dry and wet conditions, are presented in Fig. 2 . The corresponding tensile properties of the membranes are summarized in Table 1. Significant differences were found for pure CHT and CHT/BG-NP composite membranes when comparing dry and wet conditions.

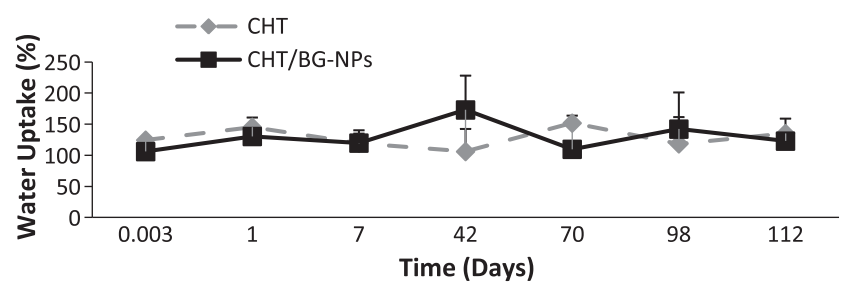

Fig. 1. Water uptake evaluation of the CHT/BG-NP composite and the pure CHT membranes over a period of 16 weeks.
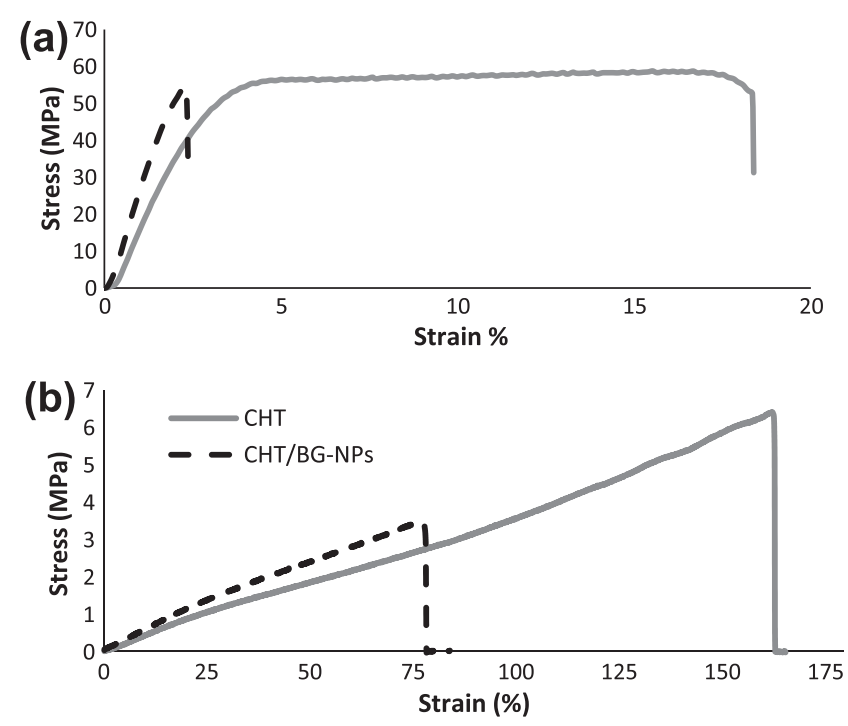

Fig. 2. Stress-strain representative curves of pure CHT and CHT/BG-NP composite membranes in (a) dry and (b) wet conditions.

In wet conditions both the Young's modulus and UTS decreased significantly compared with the dry state for both types of membrane. On the other hand, in the same conditions the elongation at failure increases by around one order of magnitude. Silva et al. also found large decreases in the secant modulus and stress at failure for pure CHT membranes, and a large increase in strain at failure upon hydration [31]. In others studies the stiffness of similar CHT membranes systematically decreased with increasing humidity, with reduction by a factor of greater than 50 relative to the dry state $[39,40]$. On the addition of BG-NPs to CHT membranes an increase in stiffness was observed under both dry and wet conditions. The strength of the membrane and the elongation at failure also tend to decrease with the presence of BG-NPs under both environmental conditions. Xianmiao et al. [38] and Teng et al. [41] performed similar studies to evaluate the properties of nanohydroxyapatite/CHT membranes, showing that the addition of nanohydroxyapatite to CHT decreased both the tensile strength and elongation at failure, but increased the elastic modulus. The membranes were much more flexible under wet conditions. Even with the introduction of an inorganic phase the composite membranes could sustain a deformation of greater than $70 \%$ in wet conditions.

\subsection{In vitro bioactivity tests}

In this study nanoparticles were used in the preparation of the composites (see inset in Fig. 3b). As shown in the inset in Fig. 3b the BG-NPs have a spherical, somewhat interconnected appearance, with particle sizes in the range $30-50 \mathrm{~nm}$.

In vitro bioactivity tests were performed on both the pure CHT and composite membranes. No apatite layer formed on the CHT membrane surface after soaking in SBF for 5 days (see Fig. 3a). Before soaking in SBF the surface of the composite membrane containing 30\% BG-NPs showed some protuberances that could indicate some agglomeration of the BG-NPs (see Fig. 3b). The bioactive potential of the composite membranes produced was confirmed by the development of an apatite layer after immersion in SBF for 5 days (see SEM image in Fig. 3c). Fig. 3d shows needlelike crystals arranged in typical cauliflower-like clusters.

The EDX data presented in Fig. 4 shows that the silicon (Si) content of the composite membranes decreased after 5 days in SBF, followed by an increase in calcium (Ca) and phosphorous (P). The increases in Ca and $\mathrm{P}$, accompanied by a decrease in the 
Table 1

Mechanical properties of pure CHT membranes and CHT/BG-NP composite membranes under wet and dry conditions.

\begin{tabular}{|c|c|c|c|c|c|c|c|}
\hline \multirow[t]{2}{*}{ Membranes } & \multicolumn{2}{|c|}{ Young's modulus (MPa) } & \multicolumn{2}{|c|}{ Ultimate tensile strength (MPa) } & \multicolumn{2}{|c|}{ Elongation at break (\%) } & \multirow[t]{2}{*}{ Water uptake (\%) } \\
\hline & Dry & Wet & Dry & Wet & Dry & Wet & \\
\hline $\mathrm{CHT}$ & $1858 \pm 82$ & $4.0 \pm 0.4$ & $56.7 \pm 3.9$ & $6.3 \pm 0.8$ & $16.8 \pm 5.3$ & $153.2 \pm 18.7$ & $145 \pm 36$ \\
\hline CHT/BG-NP & $2639 \pm 212$ & $4.7 \pm 0.3$ & $49.6 \pm 9.2$ & $3.3 \pm 0.6$ & $2.5 \pm 0.6$ & $71.6 \pm 11.6$ & $130 \pm 9$ \\
\hline
\end{tabular}

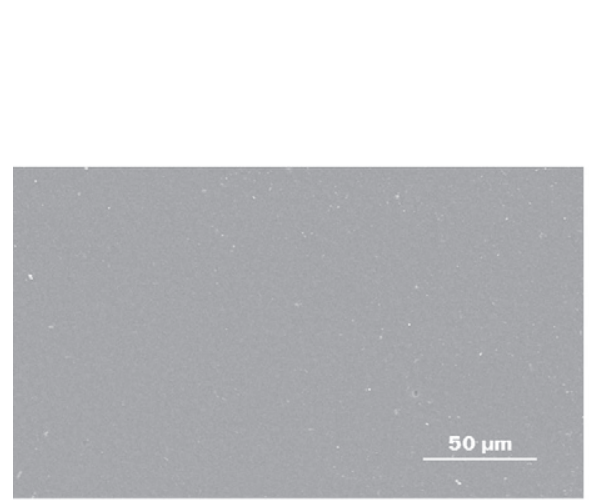

(a)

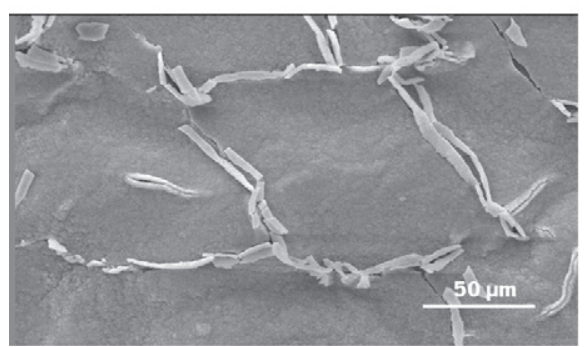

(c)

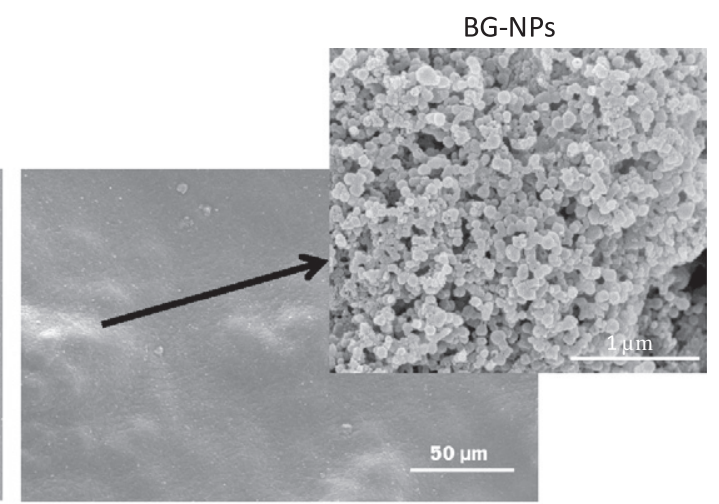

(b)

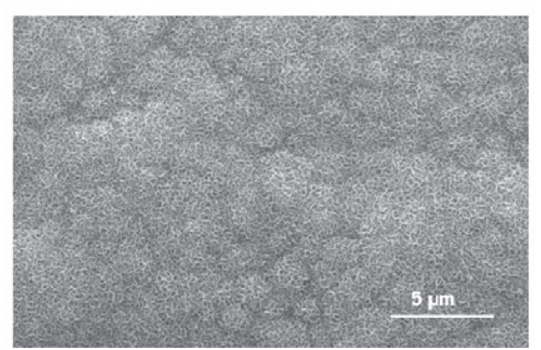

(d)

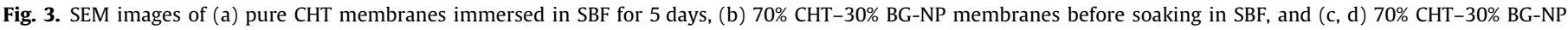
membranes immersed in SBF for 5 days at low and high magnification, respectively. The inset in (b) shows the morphology of the BG-NPs.

concentration of Si, support the indications of the development of apatite, being consistent with the SEM observations [42].

The first reaction of bioactive glasses soaked in SBF is ion exchange, in which $\mathrm{Ca}^{2+}$ and $\mathrm{Na}^{+}$in the glass exchange with $\mathrm{H}_{3} \mathrm{O}^{+}$ in the solution, resulting in a solution $\mathrm{pH}$ increase as well as in the formation of a hydrated silica gel layer. The formation of this

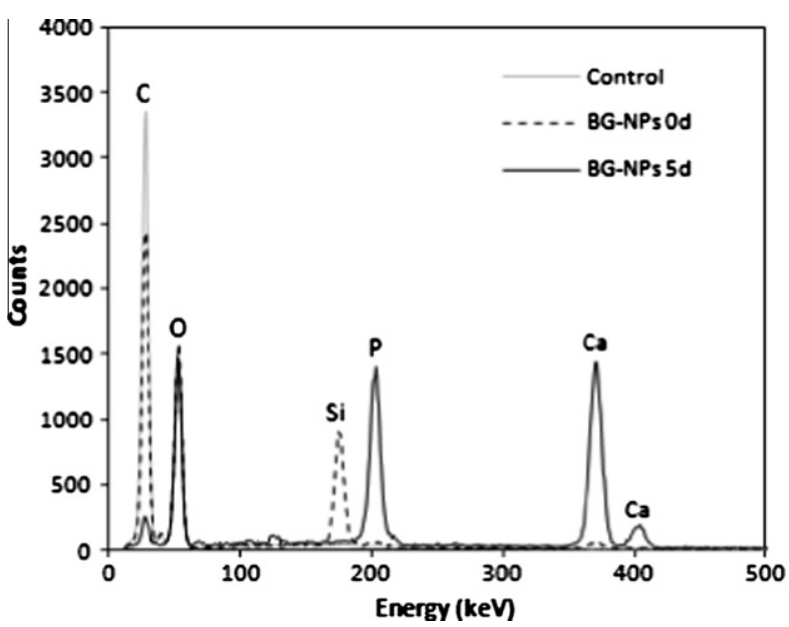

Fig. 4. EDX of the pure CHT and of the $70 \%$ CHT-30\% BG-NP membranes before and after 5 days immersion in SBF. hydrated silica gel layer on the surface of bioactive glasses which present abundant silanol $(\mathrm{Si}-\mathrm{OH})$ groups provides favorable sites for calcium phosphate nucleation. Furthermore, the water molecules in SBF react with $\mathrm{Si}-\mathrm{O}-\mathrm{Si}$ bonds to form additional $\mathrm{Si}-\mathrm{OH}$ groups. These functional groups then induce apatite nucleation, which is accelerated by the released $\mathrm{Ca}^{2+}$ and $\mathrm{Na}^{+}$ions increasing the ionic activity product of apatite in the fluid. The in vitro bioactivity results are in agreement with previous data reporting the osteoconductive character of composites containing bioactive glass/ceramic particles [11,24,28,42]. Kong et al. [21] studied the bioactivity of $\mathrm{CHT} /$ nanohydroxyapatite composite scaffolds and concluded that the addition of nanohydroxyapatite enhanced the bone bioactivity of CHT scaffolds.

\subsection{DNA content and fluorescence staining assay}

Preliminary cytotoxicity tests were performed with hPDL on pure CHT and CHT/BG-NP composite membranes, which confirmed that neither type of membrane was cytotoxic (data not shown). The presence of cells on pure CHT and CHT/BG-NPs composite membranes was demonstrated by a fluorescence staining test (Fig. 5). Cell proliferation on both types of membranes was evaluated using the PicoGreen assay (Fig. 6).

The DNA concentration associated with cell proliferation showed that hBMSC presented lower proliferation compared with hPDL. Such a difference is also observed during the expansion of 
both types of cell in culture flasks. Fluorescence micrograph also showed higher numbers of hPDL than hBMSC on CHT membranes after 3 days culture. In the case of hBMSC no significant differences were observed in DNA levels between the CHT and CHT/BG-NP membranes. hPDL proliferated over time on both the CHT and CHT/BG-NP membranes. By day 28 the amount of DNA in hPDL was significantly higher for the CHT/BG-NP composite membranes. Some previous studies reporting cell proliferation on bioactive glass composites also demonstrated that the addition of bioactive glasses could promote cell proliferation [43-46].

\subsection{Alamar blue assay}

The Alamar blue assay was performed to determine the relative differences in metabolic activity between hPDL and hBMSC cultured on pure CHT and CHT/BG-NP composite membranes. Normalization was to the ratio between fluorescence intensity levels of CHT/BG-NP composite membranes and pure CHT membranes (see Fig. 7).

Generally cell metabolic activity was higher in the CHT/BG-NP group compared with the CHT group for both hPDL and hBMSC. In the case of hBMSC the ratio tended to increase over time. The Alamar blue results suggest that the metabolic activity of both kinds of cells was higher on the CHT/BG-NP composite membranes. Sun et al. [47] used the same assay to show that akermite ceramic, which is also a bioactive ceramic, promoted hBMSC proliferation. Some other studies with bioactive glasses have shown that silicon and other released products promoted the proliferation of different cell types $[45,48-50]$. Hong et al. showed that BG-NPs with similar compositions to those tested in the present study stimulated the attachment and spreading of bone marrow stem cells [24].

\subsection{Calcium content}

Calcium formation was analyzed on days 21 and 28 of cell culture on pure CHT and CHT/BG-NP composite membranes. The data

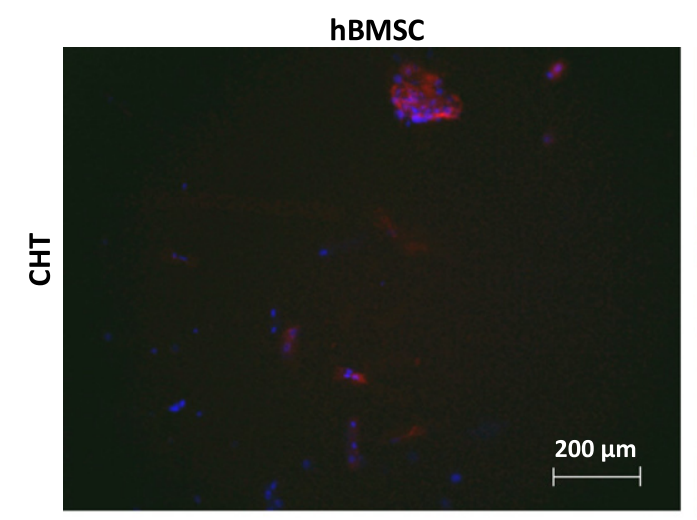

for day 28 are presented in Fig. 8, including calcium release from membranes non-seeded with cells (bare membranes).
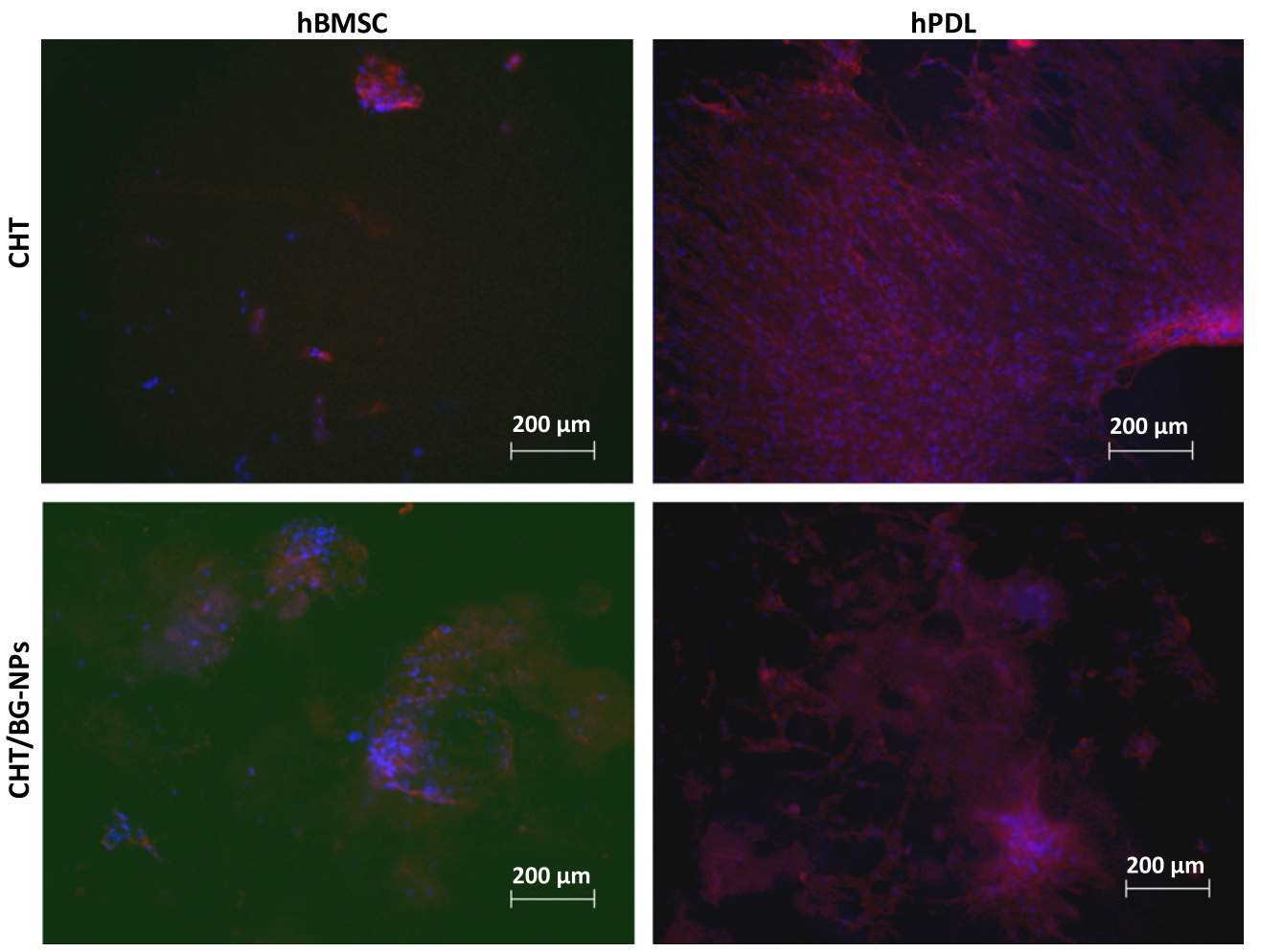

Fig. 5. Fluorescence staining assay 3 days after seeding of hBMSC and hPDL on both the CHT and CHT/BG-NP membranes.
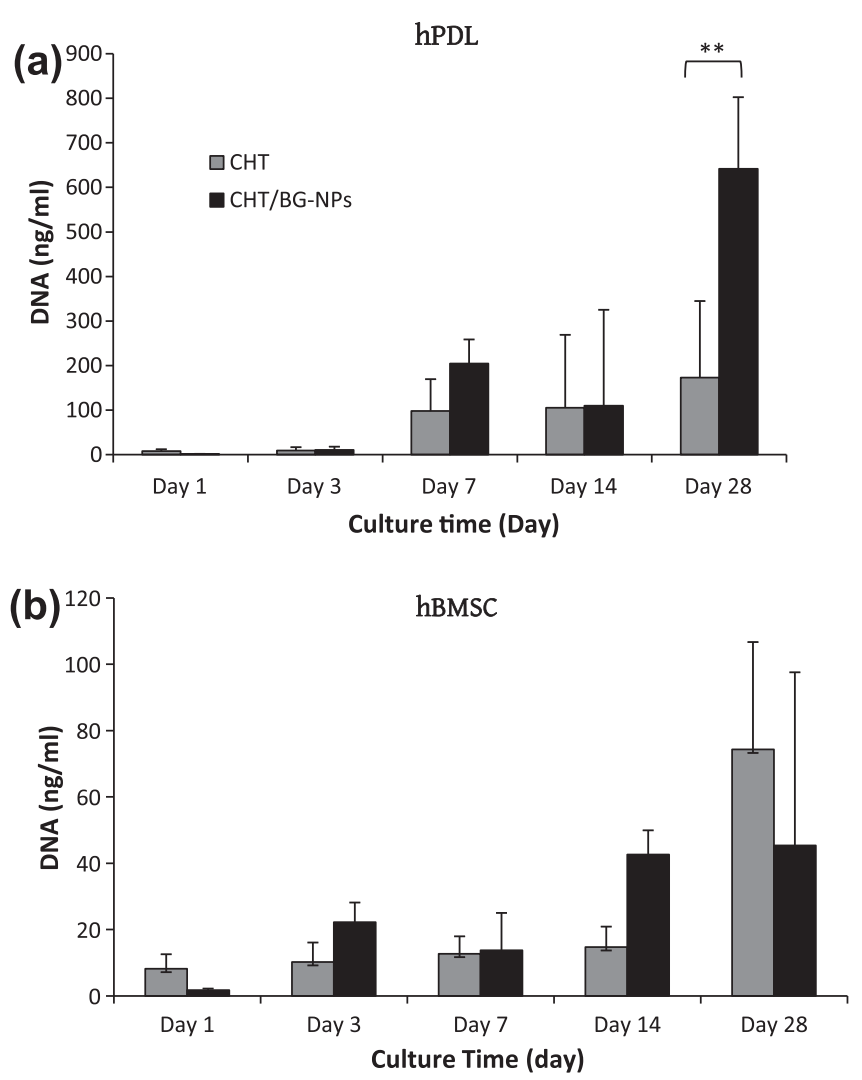

Fig. 6. DNA content measurements using (a) hPDL and (b) hBMSC cells on pure CHT and CHT/BG-NP composite membranes. ${ }^{* *} P<0.01$ 

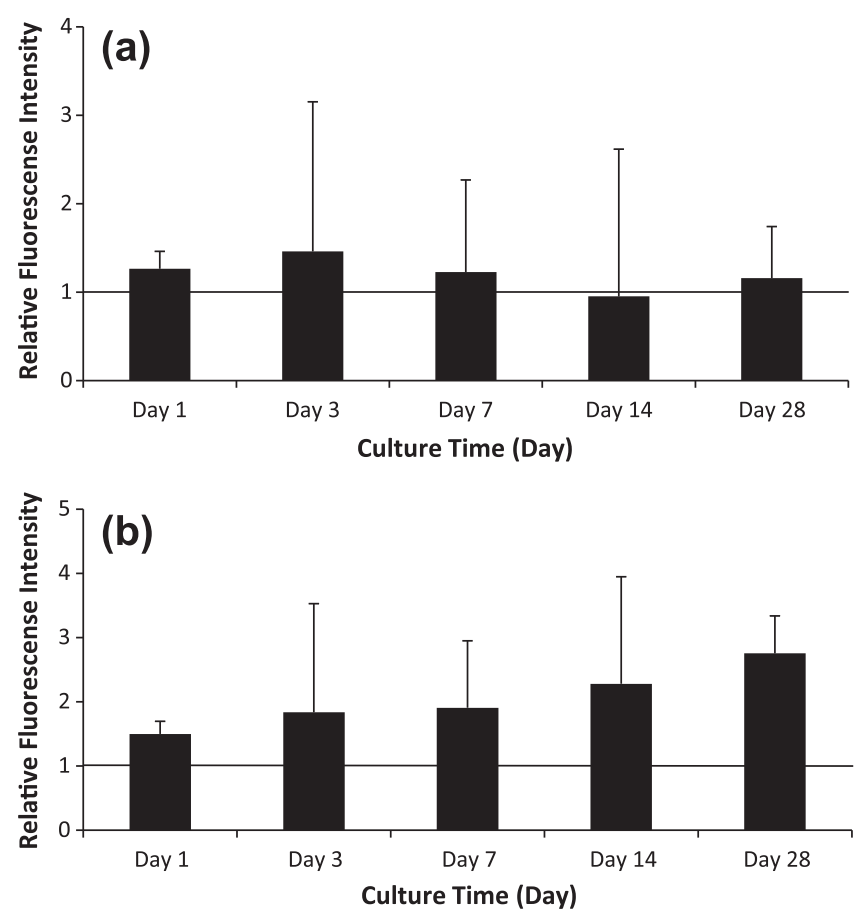

Fig. 7. Ratio of fluorescence intensity between CHT/BG-NP composite membranes and CHT membranes due to (a) hPDL and (b) hBMSC, obtained from Alamar blue assays.

The calcium concentration in the presence of hPDL and hBMSC cells on CHT/BG-NP composite membranes was significantly higher than on pure CHT membranes and control groups, indicating a positive effect of the presence of BG-NPs on cell mineralization. The highest calcium contents were found for membranes with BG-NPs, which could be attributed to the release of calcium from the inorganic nanoparticles. Dieudonné et al. [51] concluded that the high base calcium levels of bioactive glasses (in the absence of cells) could be responsible for the increased mineralization. However, in the present study the calcium levels on day 28 on the CHT/BG-NP composite membranes in the presence of cells were significantly higher than on bare composite membranes. This result indicates that the increased calcium formation could be a result of cell matrix mineralization. Others studies have demonstrated that the presence of bioactive glasses can improve cell mineralization $[11,48,52]$. On the CHT/BG-NP composite membranes both type of cell showed an increase in calcium levels between day 21 (data not shown) and day 28, indicating that mineralized matrix formation occurred over time, which means that the cells continued to mineralize after 21 days.

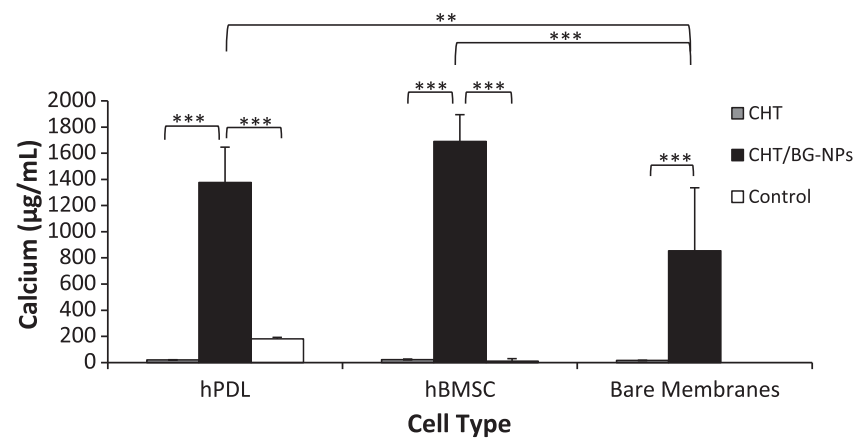

Fig. 8. Calcium content measurements of hPDL and hBMSC on CHT membranes and CHT/BG-NP composite membranes, and bare membranes. ${ }^{* * *} P<0.001 ;{ }^{* *} P<0.01$.

\section{Conclusion}

CHT/BG-NP composite membranes were studied for possible application as GTR membranes and for GBR, provided by the addition of BG-NPs to the polymer, promoting the possibility of periodontal regeneration. The introduction of BG-NPs into the CHT increased the stiffness of the membrane and the composite membranes showed adequate extensibility in wet conditions. Upon immersion in SBF the composite membranes, but not pure CHT membranes, were able to promote the deposition of an apatite layer, evidence of osteoconductive potential. The present study compared the in vitro biological performance of pure CHT membranes with CHT/BG-NP composite membranes. The metabolic activity of hPDL and hBMSC was enhanced on the addition of BGNPs to CHT membranes. An increase in cell proliferation of hPDL on CHT/BG-NP composite membranes was verified compared with pure CHT membranes. The incorporation of BG-NPs into the membrane promoted greater cell matrix mineralization by both types of cells.

The CHT/BG-NP composite membranes studied could be used as barrier membranes to prevent the invasion of periodontal defects by soft tissues, since these membranes did not show early degradation and were not cytotoxic. The results obtained suggest the possible applicability of these composite membranes in GBR and, consequently, periodontal regeneration.

\section{Acknowledgement}

This work was financially supported by the Foundation for Science and Technology (FCT) within the project PTDC/CTM-BPC/ $112774 / 2009$.

\section{Appendix A. Figures with essential colour discrimination}

Certain figures in this article, particularly Fig. 5, are difficult to interpret in black and white. The full colour images can be found in the on-line version, at http://dx.doi.org/10.1016/j.actbio.2012. 06.040.

\section{References}

[1] Friedrich D. Treatment of extended periodontal defects using a $\beta$-TCP composite material. Dent Implantol 2009;4:258-62.

[2] Buser D, Brägger U, Lang NP, Nyman S. Regeneration and enlargement of jaw bone using guided tissue regeneration. Clin Oral Implan Res 1990;1:22-32.

[3] Kay SA, Wisner-Lynch L, Marxer M, Lynch SE. Guided bone regeneration: integration of a resorbable membrane and a bone graft material. Pract Periodontics Aesthet Dent 1997;9:185-94.

[4] Zitzmann N, Naef R, Schärer P, Schüpbach P. Guided bone regeneration and augmentation in implant surgery, using Bio-Oss together with the membrane technique. Dtsch Zahnarztl Z 1996;6:51.

[5] Lanza RP, Langer RS, Vacanti J. Principles of tissue engineering. New York: Elsevier Academic Press; 2007.

[6] Bunyaratavej P, Wang HL. Collagen membranes: a review. J Periodontol 2001;72:215-29.

[7] Alves NM, Mano JF. Chitosan derivatives obtained by chemical modifications for biomedical and environmental applications. Int J Biol Macromol 2008;43:401-14.

[8] Chatelet C, Damour O, Domard A. Influence of the degree of acetylation on some biological properties of chitosan films. Biomaterials 2001;22:261-8.

[9] Lahiji A, Sohrabi A, Hungerford DS, Frondoza CG. Chitosan supports the expression of extracellular matrix proteins in human osteoblasts and chondrocytes. J Biomed Mater Res 2000;51:586-95.

[10] Li Z, Ramay HR, Hauch KD, Xiao D, Zhang M. Chitosan-alginate hybrid scaffolds for bone tissue engineering. Biomaterials 2005;26:3919-28.

[11] Lu HH, El-Amin SF, Scott KD, Laurencin CT. Three-dimensional, bioactive biodegradable, polymer-bioactive glass composite scaffolds with improved mechanical properties support collagen synthesis and mineralization of human osteoblast-like cells in vitro. J Biomed Mater Res A 2003;64:465-74.

[12] Mi F-L, Shyu S-S, Wu Y-B, Lee S-T, Shyong J-Y, Huang R-N. Fabrication and characterization of a sponge-like asymmetric chitosan membrane as a wound dressing. Biomaterials 2001;22:165-73. 
[13] Zhang M, Li XH, Gong YD, Zhao NM, Zhang XF. Properties and biocompatibility of chitosan films modified by blending with PEG. Biomaterials 2002;23: 2641-8.

[14] Park S-B, You J-O, Park H-Y, Haam SJ, Kim W-S. A novel pH-sensitive membrane from chitosan - TEOS IPN; preparation and its drug permeation characteristics. Biomaterials 2001;22:323-30.

[15] Stavropoulos A, Sculean A, Karring T. GTR treatment of intrabony defects with PLA/PGA copolymer or collagen bioresorbable membranes in combination with deproteinized bovine bone (Bio-Oss). Clin Oral Invest 2004;8:226-32.

[16] Yamaguchi I, Tokuchi K, Fukuzaki H, Koyama Y, Takakuda K, Monma H, et al. Preparation and microstructure analysis of chitosan/hydroxyapatite nanocomposites. J Biomed Mater Res 2001;55:20-7.

[17] Wan Y, Creber KAM, Peppley B, Bui VT. Ionic conductivity of chitosan membranes. Polymer 2003;44:1057-65.

[18] Park YJ, Lee YM, Lee JY, Seol YJ, Chung CP, Lee SJ. Controlled release of plateletderived growth factor-BB from chondroitin sulfate-chitosan sponge for guided bone regeneration. J Control Release 2000;67:385-94.

[19] Seol Y-J et al. Chitosan sponges as tissue engineering scaffolds for bone formation. Biotechnol Lett 2004;26:1037-41.

[20] Zhang Y, Zhang M. Synthesis and characterization of macroporous chitosan/ calcium phosphate composite scaffolds for tissue engineering. J Biomed Mater Res 2001;55:304-12

[21] Kong L, Gao Y, Lu G, Gong Y, Zhao N, Zhang X. A study on the bioactivity of chitosan/nano-hydroxyapatite composite scaffolds for bone tissue engineering. Eur Polym J 2006;42:3171-9.

[22] Boccaccini AR, Erol M, Stark WJ, Mohn D, Hong Z, Mano JF. Polymer/bioactive glass nanocomposites for biomedical applications: a review. Compos Sci Technol 2010;70:1764-76

[23] Boccaccini AR, Blaker JJ, Maquet V, Chung W, Jérôme R, Nazhat SN. Poly(D,Llactide) (PDLLA) foams with $\mathrm{TiO}_{2}$ nanoparticles and $\mathrm{PDLLA} / \mathrm{TiO}_{2}$-Bioglass ${ }^{\circledR}$ foam composites for tissue engineering scaffolds. J Mater Sci 2006;41: 3999-4008.

[24] Hong Z, Luz GM, Hampel PJ, Jin M, Liu A, Chen X, et al. Mono-dispersed bioactive glass nanospheres: preparation and effects on biomechanics of mammalian cells. J Biomed Mater Res A 2010;95A:747-54.

[25] Hong Z, Merino EG, Reis RL, Mano JF. Novel rice-shaped bioactive ceramic nanoparticles. Adv Eng Mater 2009;11:B25-9.

[26] Zhitomirsky D, Roether JA, Boccaccini AR, Zhitomirsky I. Electrophoretic deposition of bioactive glass/polymer composite coatings with and without HA nanoparticle inclusions for biomedical applications. J Mater Process Technol 2009;209:1853-60.

[27] Luz GM, Mano JF. Preparation and characterization of bioactive glass nanoparticles prepared by sol-gel for biomedical applications. Nanotechnology 2011;22:11.

[28] Hong Z, Reis RL, Mano JF. Preparation and in vitro characterization of novel bioactive glass ceramic nanoparticles. J Biomed Mater Res A 2009;88A: 304-13.

[29] Nordtveit RJ, Varum KM, Smidsrod O. Degradation of partially N-acetylated chitosans with hen egg white and human lysozyme. Carbohydr Polym 1996;29:163-7.

[30] Kokubo T, Takadama H. How useful is SBF in predicting in vivo bone bioactivity? Biomaterials 2006;27:2907-15.

[31] Silva RM, Silva GA, Coutinho OP, Mano JF, Reis RL. Preparation and characterisation in simulated body conditions of glutaraldehyde crosslinked chitosan membranes. J Mater Sci Mater M 2004;15:1105-12.

[32] Ren D, Yi H, Wang W, Ma X. The enzymatic degradation and swelling properties of chitosan matrices with different degrees of $\mathrm{N}$-acetylation. Carbohydr Res 2005;340:2403-10.

[33] Peter M, Binulal NS, Nair SV, Selvamurugan N, Tamura H, Jayakumar R. Novel biodegradable chitosan-gelatin/nano-bioactive glass ceramic composite scaffolds for alveolar bone tissue engineering. Chem Eng J 2010;158:353-61.
[34] Peter M, Binulal NS, Soumya S, Nair SV, Furuike T, Tamura H, et al. Nanocomposite scaffolds of bioactive glass ceramic nanoparticles disseminated chitosan matrix for tissue engineering applications. Carbohydr Polym 2010;79:284-9.

[35] Maquet V, Boccaccini AR, Pravata L, Notingher I, Jerome R. Porous poly(alphahydroxyacid)/Bioglass composite scaffolds for bone tissue engineering. I: Preparation and in vitro characterisation. Biomaterials 2004;25:4185-94.

[36] Maquet V, Boccaccini AR, Pravata L, Notingher I, Jérôme R. Preparation, characterization, and in vitro degradation of bioresorbable and bioactive composites based on Bioglass ${ }^{\circledR}$-filled polylactide foams. J Biomed Mater Res A 2003;66A:335-46.

[37] Bottino MC, Thomas V, Janowski GM. A novel spatially designed and functionally graded electrospun membrane for periodontal regeneration. Acta Biomater 2011;7:216-24.

[38] Xianmiao C, Yubao L, Yi Z, Li Z, Jidong L, Huanan W. Properties and in vitro biological evaluation of nano-hydroxyapatite/chitosan membranes for bone guided regeneration. Mater Sci Eng C 2009;29:29-35.

[39] Caridade SG, da Silva RMP, Reis RL, Mano JF. Effect of solvent-dependent viscoelastic properties of chitosan membranes on the permeation of 2phenylethanol. Carbohydr Polym 2009;75:651-9.

[40] Mano JF. Viscoelastic properties of chitosan with different hydration degrees as studied by dynamic mechanical analysis. Macromol Biosci 2008;8:69-76.

[41] Teng SH, Lee EJ, Yoon BH, Shin DS, Kim HE, Oh JS. Chitosan/ nanohydroxyapatite composite membranes via dynamic filtration for guided bone regeneration. J Biomed Mater Res A 2009;88:569-80.

[42] Hong Z, Reis RL, Mano JF. Preparation and in vitro characterization of scaffolds of poly(L-lactic acid) containing bioactive glass ceramic nanoparticles. Acta Biomater 2008;4:1297-306.

[43] Au AY, Au RY, Al-Talib TK, Eves B, Frondoza CG. Consil ${ }^{\circledR}$ bioactive glass particles enhance osteoblast proliferation and maintain extracellular matrix production in vitro. J Biomed Mater Res A 2008;86A:678-84.

[44] Demirkiran H, Mohandas A, Dohi M, Fuentes A, Nguyen K, Aswath P. Bioactivity and mineralization of hydroxyapatite with bioglass as sintering aid and bioceramics with $\mathrm{Na}_{3} \mathrm{Ca}_{6}\left(\mathrm{PO}_{4}\right)_{5}$ and $\mathrm{Ca}_{5}\left(\mathrm{PO}_{4}\right)_{2} \mathrm{SiO}_{4}$ in a silicate matrix. Mater Sci Eng C 2010;30:263-72.

[45] Valerio P, Pereira MM, Goes AM, Leite MF. The effect of ionic products from bioactive glass dissolution on osteoblast proliferation and collagen production. Biomaterials 2004;25:2941-8.

[46] Verrier S, Blaker JJ, Maquet V, Hench LL, Boccaccini AR. PDLLA/Bioglass ${ }^{\circledR}$ composites for soft-tissue and hard-tissue engineering: an in vitro cell biology assessment. Biomaterials 2004;25:3013-21.

[47] Sun H, Wu C, Dai K, Chang J, Tang T. Proliferation and osteoblastic differentiation of human bone marrow-derived stromal cells on akermanitebioactive ceramics. Biomaterials 2006;27:5651-7.

[48] Gough JE, Jones JR, Hench LL. Nodule formation and mineralisation of human primary osteoblasts cultured on a porous bioactive glass scaffold. Biomaterials 2004;25:2039-46.

[49] Silver IA, Deas J, Erecińska M. Interactions of bioactive glasses with osteoblasts in vitro: effects of $45 \mathrm{~S} 5$ Bioglass $^{\circledR}$, and $58 \mathrm{~S}$ and $77 \mathrm{~S}$ bioactive glasses on metabolism, intracellular ion concentrations and cell viability. Biomaterials 2001;22:175-85.

[50] Silver IA, Erecinska M. Interactions of osteoblastic and other cells with bioactive glasses and silica in vitro and in vivo. Materialwiss Werkst 2003;34:1069-75.

[51] Dieudonné SC et al. Osteoblast differentiation of bone marrow stromal cells cultured on silica gel and sol-gel-derived titania. Biomaterials 2002;23: 3041-51.

[52] Jones JR, Tsigkou O, Coates EE, Stevens MM, Polak JM, Hench LL. Extracellular matrix formation and mineralization on a phosphate-free porous bioactive glass scaffold using primary human osteoblast (HOB) cells. Biomaterials 2007;28:1653-63. 